\title{
Prevalence of Physical Frailty: Results from the DO-HEALTH Study
}

\author{
M. Gagesch ${ }^{1,2}$, P.O. Chocano-Bedoya ${ }^{2,3}$, L.A. Abderhalden ${ }^{1,2}$, G. Freystaetter ${ }^{1,2}$, A. Sadlon ${ }^{1,2}$, J.A. Kanis ${ }^{4}$, \\ R.W. Kressig ${ }^{5}$, S. Guyonnet ${ }^{6}$, J.A.P. DaSilva ${ }^{7}$, D. Felsenberg ${ }^{8}$, R. Rizzoli ${ }^{9}$, M. Blauth ${ }^{10}$, E.J. Orav ${ }^{11}$, A. Egli ${ }^{2}$, \\ H.A. Bischoff-Ferrari ${ }^{1,2,12}$
}

\begin{abstract}
1. Department of Geriatrics, University Hospital Zurich, Zurich, Switzerland; 2. Centre on Aging and Mobility, University of Zurich, Zurich, Switzerland; 3. Institute of Primary Health Care (BIHAM), University of Bern, Bern, Switzerland; 4. Mary McKillop Institute for Health Research, Australian Catholic University, Melbourne, Australia and Centre for Metabolic Bone Diseases, University of Sheffield Medical School, Sheffield, UK; 5. University Department of Geriatric Medicine FELIX PLATTER, Basel, Switzerland; 6. Gérontopôle, Department of Geriatrics, CHU Toulouse, Toulouse, France; 7. Institute for Clinical and Biomedical Research (i.CBR), Faculty of Medicine, University of Coimbra, Department of Rheumatology, Centro Hospitalar e Universitário de Coimbra, Coimbra, Portugal; 8. Centre for Muscle and Bone Research, Department of Radiology, Charité Universitätsmedizin Berlin, Berlin, Germany (Prof. Felsenberg is deceased); 9. Service of Bone Diseases, Geneva University Hospitals and Faculty of Medicine, Geneva, Switzerland; 10. University of Innsbruck, Innsbruck, Austria; 11. Department of Medicine, Harvard Medical School, Boston, MA, USA; 12. University Clinic for Acute Geriatric Care, City Hospital Waid and Triemli, Zurich, Switzerland
\end{abstract}

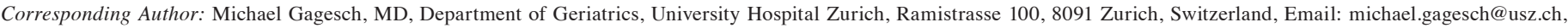
https://orcid.org/0000-0003-3089-5768

\begin{abstract}
BACKGROUND: Frailty is a geriatric syndrome associated with multiple negative health outcomes. However, its prevalence varies by population and instrument used. We investigated frailty and prefrailty prevalence by 5 instruments in community-dwelling older adults enrolled to a randomized-controlled trial in 5 European countries.

METHODS: Cross-sectional baseline analysis in 2,144 DO-HEALTH participants recruited from Switzerland, Austria, France, Germany, and Portugal with complete data for frailty. Frailty status was assessed by the Physical Frailty Phenotype [PFP], SOF-Frailty Index [SOF-FI], FRAIL-Scale, SHARE-Frailty Instrument [SHARE-FI], and a modified SHARE-FI, and compared by country, age, and gender. Logistic regression was used to determine relevant factors associated with frailty and pre-frailty.

RESULTS: Mean age was $74.9( \pm 4.4)$ years, $61.6 \%$ were women. Based on the PFP, overall frailty and pre-frailty prevalence was $3.0 \%$ and $43.0 \%$. By country, frailty prevalence was highest in Portugal $(13.7 \%)$ and lowest in Austria (0\%), and pre-frailty prevalence was highest in Portugal (57.3\%) and lowest in Germany (37.1\%). By instrument and overall, frailty and pre-frailty prevalence was highest based on SHAREFI $(7.0 \% / 43.7 \%)$ and lowest based on SOF-FI (1.0\% / 25.9\%). Frailty associated factors were residing in Coimbra (Portugal) [OR 12.0, CI 5.30-27.21], age above 75 years [OR 2.0, CI 1.17-3.45], and female gender [OR 2.8, CI 1.48-5.44]. The same three factors predicted prefrailty.

CONCLUSIONS: Among relatively healthy adults age 70 and older enroled to DO-HEALTH, prevalence of frailty and pre-frailty differed significantly by instrument, country, gender, and age. Among instruments, the highest prevalence of frailty and pre-frailty was documented by the SHARE-FI and the lowest by the SOF-FI.
\end{abstract}

Key words: Frailty, community-dwelling, prevalence, clinical trials, epidemiology.

\section{Introduction}

$\mathrm{F}$ Trailty is an age-related medical syndrome of reduced functional capacity and one of the most burdensome conditions for the growing segment of the older population, and society as a whole (1). Frailty is often preceded by sarcopenia (2), the age-associated loss of muscle mass and function and both concepts overlap, e.g. in regard to reduced muscle strength, physical function (e.g. gait speed), and unintentional weight loss (3). In addition, frailty and sarcopenia are often promoted by factors such as malnutrition $(4,5)$ and multimorbidity $(6,7)$.

Over the last two decades, frailty has been identified as a stronger predictor of acute-care complications, length of stay, and hospital readmission than multimorbidity or chronological age alone (8-11). E.g. in a 2016 systematic review and metaanalysis of 31 prospective studies, Vermeiren et al. found that frailty increases the likelihood of mortality by 1.8-2.3fold, hospital admissions by 1.2-1.8-fold, incident functional impairment related to basic activities of daily living by $1.6-2.0$, and falls and fractures by 1.2-2.8-fold (12).

The development of frailty in older adults should be recognized as a dynamic process including transitions from robust, to pre-frail and to frail and vice versa over time (13). Therefore, early detection of the at-risk group of prefrail individuals appears as a window of opportunity for the prevention of overt frailty, and perhaps prevention of sarcopenia as well $(3,14,15)$.

While the identification of frailty is becoming increasingly recognized as important, the choice of frailty instrument might be guided by medical practice within a specialty, the population, setting, and outcome under investigation (16). Frailty is actively screened for in various clinical settings, including oncology and surgical specialties. However, the lack of consensus for defining frailty still hinders the comparison of frailty prevalence between different populations (1, 17-19).

Over time, the concept of the Physical Frailty Phenotype (PFP), introduced by Fried and colleagues in 2001 (20) and the deficit accumuation approach (i.e. Frailty Index) introduced by Mitnitski and Rockwood $(21,22)$ have emerged as the most frequently cited ways to operationalize frailty (23). While the Frailty Index approach scores accumulating deficits in age-associated variables covering multipe body systems (24), the five components of the PFP (fatigue, weight loss, 
slowness, weakness, and low activity level) aggregate to a clinical syndrome (20). Subsequently, many researchers have modified the PFP in order to accommodate variables available from research studies' data sets (25). Among those, the Study of Osteoporotic Fractures (SOF)-Frailty Index (26), a condensed variation of only 3 items, and the FRAIL-Scale (27), a selfreport based 5-item screening tool substituting the low activity criterion with multimorbidity (defined as the presence of five or more chronic diseases) have been frequently cited.

To our knowledge, the prevalence of frailty and pre-frailty in older Europeans according to the PFP has not been studied in a single multi-national dataset with the exception of the Survey of Health Aging and Retirement in Europe (SHARE) database in 2009 and $2019(28,29)$. The reported frailty prevalence by the latter study in SHARE ranged from 3.0\% (Switzerland) to $15.6 \%$ (Portugal), thus indicating an uneven distribution in the older European population, plus indicating a need for further studies on frailty and its assessment concepts in older Europeans.

DO-HEALTH is a clinical trial in relatively healthy community-dwelling participants age 70 years and older without major health events (cancer or myocardial infarction) in the 5 years prior to enrollment, sufficient mobility to come to the study centers without help, and intact cognitive function, recruited from 5 European countries (30). Examining the prevalence of frailty and pre-frailty in DO-HEALTH at baseline aims to contribute important insights to the participants' clinical presentation upon enrolment, and also with regard to several primary endpoints of the study, including cardiovascular health and physical function (30).

In view of the variety of instruments derived from the PFP, and to estimate the variability of frailty prevalence based on the instrument used, we investigated the PFP as our primary measure and four alternative derived instruments in DO-HEALTH. However, one has to acknowledge that this is not a probabilisitc sample of the countries investigated: in addition to the DO-HEALTH eligibility criteria listed above, each countries sample consisted of older adults volunteering to participate in a clinical trial, and recruited according to the methods of each study site.

In addition to examine the variability in frailty according to instrument, we examine whether the prevalence of frailty and pre-frailty differs by country, age, and gender.

\section{Materials and methods}

This is a cross-sectional study taking advantage of the baseline examination of 2,144 of 2,157 participants of the DO-HEALTH clinical trial recruited from Switzerland (Zurich, Basel, Geneva), Austria (Innsbruck), France (Toulouse), Germany (Berlin), and Portugal (Coimbra), with complete data for frailty. As a multicenter randomized controlled trial (RCT), DO-HEALTH (clinical trials: NCT01745263) was designed to investigate the effect of Vitamin D3 and Omega 3-fatty acids supplementation and a simple home exercise program in a $2 \times 2 \times 2$ factorial design over a three year follow-up addressing multiple endpoints associated with aging, including frailty (30).
Inclusion criteria for DO-HEALTH were age 70 years and older, a Mini-Mental Status Examination Score of at least 24 points (31), living in the community and being sufficiently mobile to visit the study center, i.e. being capable to walk 10 meters and getting in and out of a chair without help. In order to capture the broad scope of the trial's endpoints, the participants were meticulously phenotyped with standardized assessments of multiple organ, cognitive, and physical function at each of the four whole-day clinical study visits (baseline and follow-up at 12, 24, and 36 months). Assessments followed a strict protocol with regular site visits for inter-center quality control, overseen by the primary investigator. Further details of the design, aims and scope, exclusion criteria, and primary endpoints of DO-HEALTH are described elsewhere (30).

\section{Assessments of Physical Function and Comorbidities}

For the assessment of physical function, we used the Short Physical Performance Battery (SPPB), a validated assessment tool for lower extremity function in older adults (32). The SPPB includes a gait speed test, a chair-rise test, and a balance test. Its three components each score $0-4$, with 4 points indicating the highest level of performance, and add up to an overall score of maximum 12 points. The number of comorbidities was recorded by a self-administered comorbidity questionnaire (33).

\section{Fried Physical Frailty Phenotype}

The physical frailty phenotype (PFP) has been validated in community dwelling older adults from numerous countries and in many in- and outpatient settings by various operationalizations $(17,25,34)$. We built our PFP model with variables available from the baseline DO-HEALTH dataset as our primary frailty assessment tool. Unintentional weight loss was defined as a self-reported loss of more than $5 \%$ of body weight over the prior 12 months. Fatigue was operationalized as a positive answer to the self-reported question: "In the last month, have you had too little energy to do things you wanted to?" from the SHARE study original questionnaire. For weakness, grip-strength was taken in kilopascal $(\mathrm{kPa})$ from the best of three consecutive trials at the dominant hand using a Martin Vigorimeter (KLS Martin Group, Tuttlingen, Germany) (35). Across all countries and ages, we used cut points by the lowest quintile approach by gender as did Fried and colleagues in their landmark study, but for simplification, irrespective of BMI (20). Slowness was defined as a gait speed below 0.67 $\mathrm{m} / \mathrm{s}$ and $0.7 \mathrm{~m} / \mathrm{s}$ respectively, according to gender and height as in the original study by Fried et al (20). Low activity level was conceptualized as done earlier by Santos-Eggimann and colleagues analyzing the SHARE dataset (28). An answer of "less than once a week" to the question: "How often do you engage in activities that require a low or moderate level of energy such as gardening, cleaning the car, or doing a walk?" fulfilled the criterion. Pre-frailty was fulfilled with 1-2 positive criteria and frailty with $\geq 3$ positive criteria. 


\section{SOF-Frailty Index}

The Study of Osteoporotic Fractures-Frailty Index (SOF-FI), introduced by Ensrud et al. is a condensed three-item variant of the Fried PFP including only the variables for weakness, involuntary weight loss, and fatigue $(26,36)$. It has been validated in community dwelling participants in the MOBILIZE Boston Study (37). In DO-HEALTH, we used data from the chair-rise test of the Short Physical Performance Battery for the SOF-FI weakness criterion (38). Weight loss was defined by a self-reported loss of more than $5 \%$ of body weight over the prior 12 months. Fatigue was defined by a positive answer to the question: "Do you feel full of energy?" from the Geriatric Depression Scale as in the original study (26). Pre-frailty was defined by the presence of one, and frailty by $\geq 2$ positive criteria.

\section{FRAIL-Scale}

The FRAIL-Scale (Fatigue, Resistance, Ambulation, Illnesses, and Loss of Weight-Scale) consists completely of self-reported items (39). Since its introduction, it has been validated for the prediction of most relevant frailty associated negative outcomes in various settings (40-42). For building the FRAIL-Scale in DO-HEALTH, we used the same question for the fatigue criterion as described above for the PFP. For resistance (weakness) we used the question for difficulties climbing stairs from SHARE-FI (see below). For the ambulation criterion, we used the question: "Because of a health problem, do you have difficulty (expected to last more than 3 months) walking 100 meters", also from SHARE-FI. Illnesses (i.e. multimorbidity) was defined by the presence of $>5$ diagnoses). Weight loss was defined by a self-reported loss of more than $5 \%$ of body weight over the prior 12 months. On the FRAIL-Scale, pre-frailty was defined by 1-2 positive criteria, and frailty by $\geq 3$ positive criteria.

\section{SHARE-Frailty Instrument (SHARE-FI1)}

To match the available variables from DO-HEALTH, we used a marginally modified version of the original SHAREFrailty Instrument (SHARE-FI), validated in community dwelling older adults and in primary care.[43-45] Weight loss was defined by a self-reported $>5 \%$ loss of body weight over the prior 12 months. Fatigue was operationalized in the same way as for the PFP, by a positive answer to the question: "In the last month, have you had too little energy to do things you wanted to do?" For indicating low grip strength on Martin Vigorimeter readings, we used the lowest quintile approach as described for the PFP above. Slowness was defined as originally described by Santos-Eggimann et al. by a positive answer to either i) "Because of a health problem, do you have any difficulty walking 100 meters? (Exclude any difficulties that you expect to last less than three months.)", or ii) "Because of a health problem, do you have any difficulty climbing one flight of stairs without resting? (Exclude any difficulties that you expect to last less than three months.)" (28). Low activity was fulfilled by a response of "less than once a week" (i.e. "one to three times a month" or "hardly ever or never") to the same question as for the PFP: "How often do you engage in activities that require a low or moderate level of energy such as gardening, cleaning the car, or going for a walk?" Pre-frailty was defined as $1-2$ positive criteria and frailty with $\geq 3$ positive criteria. The SHARE-FI1 differs from our implementation of the PFP only in the way that slowness was assessed by selfreport only.

\section{SHARE-FI DO-HEALTH Variant (SHARE-FI2)}

For a fully self-report based variant of the SHARE-FI in DO-HEALTH, we substituted grip strength measurement by a positive answer to the question: "Because of a health problem, do you have any difficulty climbing one flight of stairs without resting (exclude any difficulties that you expect to last less than three months)?" This question was usually meant only to depict slowness in the original SHARE-FI operationalization. However, measuring resistance (or weakness) by a single question of difficulty walking one flight of stairs has been validated as a surrogate marker for weakness (46), as it is also used as the weakness criterion in the FRAIL-Scale introduced by Abellan van Kan et al. (see above) (39). For SHARE-FI2, pre-frailty was fulfilled with 1-2 positive criteria and frailty with $\geq 3$ positive criteria.

Supplementary Table 1 provides an additional overview of the single components comprising the described instruments.

\section{Statistical Analysis}

Data is reported as means and standard deviations (SD) for continuous variables and frequencies and percentages for categorical variables. We estimated the prevalence of fraily and pre-frailty as the proportion of our study population who could be classified as either frail or pre-frail according to the PFP and the derived instruments and reported as frequencies and percentages. We then compared the prevalences by country, age sub-groups $(<75 y, \geq 75 y)$, and gender using a Chi-square $(\chi 2)$ test or Fisher's exact test when a frequency was less than five. We report on the general prevalence by screening tool including participants with a maximum of two missing values, as instruments indicate frailty as having $\geq 3$ out of 5 criteria present (with the exception of the SOF where $\geq 2$ positive items indicate frailty). We constructed a logistic regression model to compare frail and non-frail patients according to the PFP by study site, age group and gender using Zurich as our reference site. A separate model was run with pre-frailty as the outcome. All analyses were performed with SAS v9.4 (SAS Inc., Carey, North Carolina, USA).

\section{Results}

Overall, mean age was $74.9 \pm 4.4$ years, and $61.6 \%$ were women. Mean Mini-Mental Status Examination score was 28.5 points (SD 1.5). Mean BMI was $26.3 \mathrm{~kg} / \mathrm{m}^{2}$ (SD 4.3), and 
Table 1. Baseline Characteristics of the DO-HEALTH participants

\begin{tabular}{|c|c|c|c|}
\hline Characteristics & $\begin{array}{c}\text { Total } \\
(n=2,157)\end{array}$ & $\begin{array}{c}\text { Men } \\
(n=826)\end{array}$ & $\begin{array}{c}\text { Women } \\
(n=1,331)\end{array}$ \\
\hline \multicolumn{4}{|l|}{ Country (study site), N (\%) } \\
\hline Switzerland & $1006(46.6)$ & $398(48.2)$ & $608(45.7)$ \\
\hline (Zurich) & $552(25.6)$ & $222(26.9)$ & $330(24.8)$ \\
\hline (Basel) & $253(11.7)$ & $102(12.4)$ & $151(11.3)$ \\
\hline (Geneva) & $201(9.3)$ & $74(9.0)$ & $127(9.5)$ \\
\hline Portugal (Coimbra) & $301(14.0)$ & $109(13.2)$ & $192(14.4)$ \\
\hline France (Toulouse) & $300(13.9)$ & $119(14.4)$ & $181(13.6)$ \\
\hline Germany (Berlin) & $350(16.2)$ & $103(12.5)$ & $247(18.6)$ \\
\hline Austria (Innsbruck) & $200(9.3)$ & $97(11.7)$ & $103(7.7)$ \\
\hline Age (years) & $74.9(4.4)$ & $75.2(4.5)$ & $74.8(4.4)$ \\
\hline BMI $\left(\mathrm{kg} / \mathrm{m}^{2}\right)$ & $26.3(4.3)$ & $26.6(3.5)$ & $26.2(4.7)$ \\
\hline MMSE Score & $28.5(1.5)$ & $28.5(1.4)$ & $28.5(1.6)$ \\
\hline Comorbiditiesa (total) & $1.7(1.4)$ & $1.5(1.4)$ & $1.9(1.4)$ \\
\hline$\geq 3$ comorbidities, $\mathrm{N}(\%)$ & $569(26.4)$ & $179(21.7)$ & $390(29.3)$ \\
\hline Gait Speed (m/s) & $1.12(0.23)$ & $1.14(0.23)$ & $1.09(0.23)$ \\
\hline Handgrip Strength (kPa) & $60.1(19.0)$ & $75.4(17.0)$ & $50.1(12.3)$ \\
\hline SPPB (sum score) & $10.9(1.5)$ & $11.0(1.4)$ & $10.7(1.6)$ \\
\hline
\end{tabular}

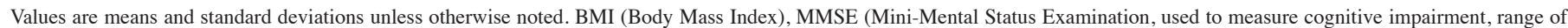

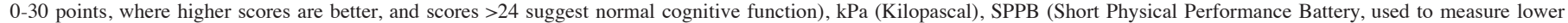
extremity function, scores range from 0-12 points, where higher scores indicate better functioning), aaccording to a Self-Administered Comorbidity Questionnaire (23).

$28.5 \%(n=569)$ of participants had three or more comorbidities at baseline. Baseline characteristics of all study participants by gender are presented in Table 1. Of the total 2,157 participants in DO-HEALTH, 2,144 (99.4\%) had complete data at baseline on at least three of the variables for all frailty measures (Supplementary Table 2). In all, 1,005 participants were included from Switzerland (1 participant was excluded from Zurich), 350 from Germany, 300 from Portugal (1 participant excluded), 289 from France (11 participants excluded), and 200 from Austria.

\section{Prevalence of frailty and pre-frailty in all DO-HEALTH participants by instrument}

According to our primary measure PFP, $3.0 \%(n=64)$ of DO-HEALTH participants were frail, and $43.0 \%(n=922)$ were pre-frail (i.e. at-risk for frailty). See Figure 1 for the overall prevalence of frailty and pre-frailty based on the PFP and the other four derived instruments. Prevalence of frailty in the total population was highest based on SHARE-FI1 (7.0\%), and lowest based on SOF-FI (1.0\%). Also, prevalence of prefrailty in the total population was highest based on SHARE-FI1 (43.7\%), and lowest based on SOF-FI (25.9\%).

\section{Prevalence of frailty and pre-frailty by country, age, and gender}

With regard to country, the prevalence of frailty was highest using the SHARE-FI1 (range 1.5-24.3\%), and lowest with the
SOF-FI (range 0-3.0\%). With regard to country, the prevalence of pre-frailty was highest with the SHARE-FI1 tool for all countries (range 40.3-51.3\%), except for Portugal (51.3\% vs. $57.3 \%$ on the PFP). With regard to country, the prevalence of pre-frailty was lowest with the SOF-FI for all countries (range 23.2-32.0\%), except for Austria (27.5\% vs. $24.0 \%$ on the FRAIL-Scale).

Figure 1. Prevalence of frailty and pre-frailty by frailty instrument among DO-HEALTH participants ( $\mathrm{n}=2,144$ with available data out of $n=2,157$ total participants)

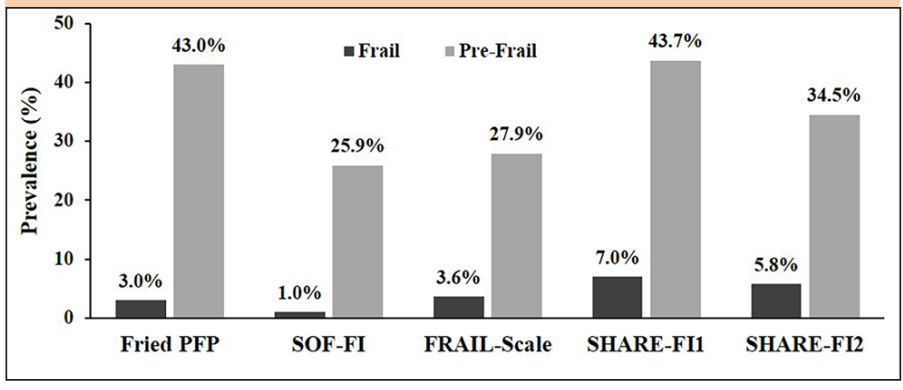

Figure 2a shows all prevalence rates for frailty by instrument and country. Figure $2 b$ shows all prevalence rates for pre-frailty by instrument and country.

With regard to age group ( $<75$ years vs. $\geq 75$ years), persons 75 years and older were more often frail compared to younger persons, regardless of the frailty instrument used $(\mathrm{P}<0.05$ for all instruments). In both age groups, the prevalence of frailty was highest on the SHARE-FI1 $(4.4 \% ; 10.4 \%, \mathrm{P}<0.0001)$ and 
lowest on the SOF-FI $(0.5 \%, 1.7 \%, \mathrm{P}=0.0069)$. With regard to age group ( $<75$ years vs. $\geq 75$ years), there were significant differences in pre-frailty with a higher proportion of pre-frail participants among those age 75 years and older $(\mathrm{P}<0.05$ for all instruments). In the younger age group, the prevalence of prefrailty was highest using the SHARE-FI1 (42.0\%), and lowest using the SOF-FI (22.9\%), while in the older age group, the prevalence of pre-frailty was highest using the PFP (46.2\%), and lowest using the SOF-FI (30.1\%).

Figure 2a. Prevalence of frailty by country and investigated frailty instrument

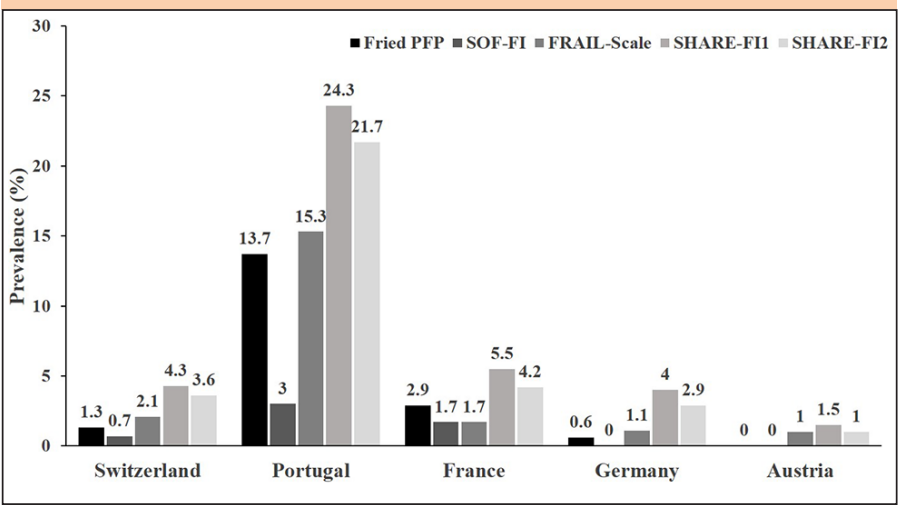

Figure $2 \mathrm{~b}$. Prevalence of pre-frailty by country and investigated frailty instrument

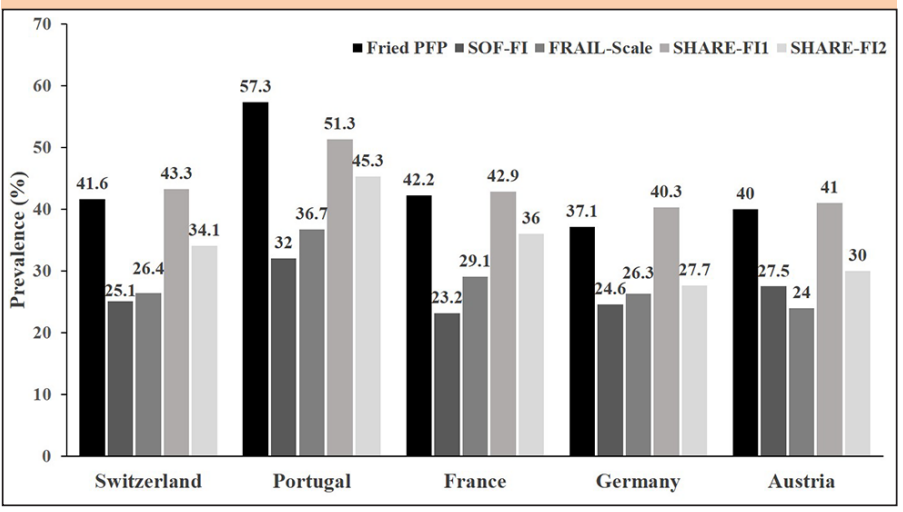

With regard to gender, there were significant differences for frailty by all investigated instruments with women having an overall higher prevalence of frailty compared to men $(\mathrm{P}<0.01)$, with the exception of the SOF-FI $(\mathrm{P}=0.3505)$. With regard to pre-frailty, there were significant differences by gender for all investigated instruments with women having an overall higher prevalence of pre-frailty compared to men $(\mathrm{P}<0.001)$.

Results for frailty by country (study site) and each subgroup are shown in detail in Table 2. Results for pre-frailty by country (study site) and each subgroup are shown in detail in Supplementary Table 3.

In our logistic regression model, we compared frail and non-frail participants according to the PFP by study center (using Zurich as our reference), age and gender. In regard to frailty, we found a 12-fold increased odds for participants from Coimbra (Portugal) [OR 12.00; CI 5.30-27.21] compared to Zurich, a 2-fold increased odds for older participants ( $\geq 75$ years of age) [OR 2.00; CI 1.17-3.45], and a 2.8-fold increased odds for female participants [OR 2.84; CI 1.48-5.44]. In regard to pre-frailty, we found an almost 2-fold increased odds for participants from Coimbra (Portugal) [OR 1.90; CI 1.432.53] compared to Zurich, a 1.2-fold increased odds for older participants ( $\geq 75$ years of age) [OR 1.20; CI 1.00-1.42], and a 1.45 -fold increased odds for female participants [OR 1.45; CI 1.21-1.73] as shown in Supplementary Table 4.

\section{Discussion}

In this large study of 2,144 relatively healthy, community dwelling European adults age 70 and older recruited from 5 European countries, and based on our primary measure PFP instrument, $3 \%$ of participants were frail and close to half of all participants were pre-frail. With regard to subgroups, prevalence of frailty and pre-frailty was highest in Portuguese participants, women, and adults age 75 and older. Notably, prevalence of frailty and pre-frailty varied substantially by the instrument used and was lowest by the SOF-FI, and highest by the SHARE-FI1.

Earlier studies in community dwelling older adults from the US and Germany reported frailty prevalence rates ranging from 2.5 to $17 \%$ using the PFP definition $(26,47-49)$. Of note, our reported frailty prevalence in DO-HEALTH with a mean age of 74.9 years was substantially lower than in similar age groups of population-based studies such as SHARE (1.3 vs. $4.4 \%$ for Switzerland, 13.8 vs. $28.6 \%$ for Portugal, 2.9 vs. $15.6 \%$ for France, 0.6 vs. $9.6 \%$ for Germany, and 0 vs. $11.6 \%$ for Austria). (29). However, the prevalence appeared more comparable to the age group between 65-74 years in the same study hereby reflecting the relatively good health of the DO-HEALH participants. At the same time our results of a higher odds for being frail and pre-frail by higher age and female gender are in line with the prior literature (1).

While our findings do not arise from a representative sample of the population, our results of a higher frailty prevalence in our Portugal center compared to the more central European countries in DO-HEALTH are consistent with the SHARE cohort findings (waves 1 and 6) and supported by a higher rate of physical inactivity in southern European countries, including Portugal $(28,29,50)$. This is further supported by a recent study investigating limitations in instrumental activities of daily living (iADL) from SHARE data, highlighting the uneven distribution of burdens in self-care with higher rates in southern European countries, including Portugal (51). Additionally, the observed health disparity with regard to frailty prevalence between Portugal and Austria or Switzerland in DO-HEALTH may be explained by a potential difference in educational level (28) and linked to national economic indicators $(52,53)$, or specific patient sampling. In fact, DO-HEALTH participants from Portugal had less years of education compared to the four other countries. Further, the 2015 OECD (Organization for Economic Co-operation and Development) report on the attainment of overall goals in health care supports our findings by ranking Switzerland (No. 2), France (No. 6), Austria (No. 10), and Germany (No. 14) relatively high, compared with Portugal (No. 
Table 2. Prevalence of Frailty by Instrument in DO-HEALTH at baseline according to country (study site), age, and gender

\begin{tabular}{|c|c|c|c|c|c|}
\hline & $\begin{array}{c}\text { Fried PFP } \\
(n=2,144)\end{array}$ & $\begin{array}{c}\text { SOF-FI } \\
(n=2,144)\end{array}$ & $\begin{array}{l}\text { FRAIL-Scale } \\
\quad(n=2,144)\end{array}$ & $\begin{array}{c}\text { SHARE-FI1 } \\
(n=2,144)\end{array}$ & $\begin{array}{l}\text { SHARE-FI2 } \\
\quad(n=2,144)\end{array}$ \\
\hline Total sample & $64(3.0)$ & $21(1.0)$ & $78(3.6)$ & $149(7.0)$ & $125(5.8)$ \\
\hline \multicolumn{6}{|l|}{ Country (study site) } \\
\hline Switzerland & $13(1.3)$ & $7(0.7)$ & $21(2.1)$ & $43(4.3)$ & $36(3.6)$ \\
\hline (Zurich) & $7(1.3)$ & $1(0.2)$ & $12(2.2)$ & $25(4.5)$ & $19(3.5)$ \\
\hline (Basel) & $4(1.6)$ & $3(1.2)$ & $7(2.8)$ & $12(4.7)$ & $14(5.5)$ \\
\hline (Geneva) & $2(1.0)$ & $3(1.5)$ & $2(1.0)$ & $6(3.0)$ & $3(1.5)$ \\
\hline Portugal (Coimbra) & $41(13.7)$ & $9(3.0)$ & $46(15.3)$ & $73(24.3)$ & $65(21.7)$ \\
\hline France (Toulouse) & $8(2.9)$ & $5(1.7)$ & $5(1.7)$ & $16(5.5)$ & $12(4.2)$ \\
\hline Germany (Berlin) & $2(0.6)$ & $0(0.0)$ & $4(1.1)$ & $14(4.0)$ & $10(2.9)$ \\
\hline Austria (Innsbruck) & $0(0.0)$ & $0(0.0)$ & $2(1.0)$ & $3(1.5)$ & $2(1.0)$ \\
\hline$P$ value & $<0.0001$ & $<0.0001$ & $<0.0001$ & $<0.0001$ & $<0.0001$ \\
\hline \multicolumn{6}{|l|}{ Age Group } \\
\hline$<75$ years & $23(1.9)$ & $6(0.5)$ & $28(2.3)$ & $54(4.4)$ & $47(3.8)$ \\
\hline$\geq 75$ years & $41(4.5)$ & $15(1.7)$ & $50(5.5)$ & $95(10.4)$ & $78(8.6)$ \\
\hline P value & 0.0004 & 0.0069 & $<0.0001$ & $<0.0001$ & $<0.0001$ \\
\hline \multicolumn{6}{|l|}{ Gender } \\
\hline Men & $12(1.5)$ & $6(0.7)$ & $17(2.1)$ & $32(3.9)$ & $23(2.8)$ \\
\hline Women & $52(3.9)$ & $15(1.1)$ & $61(4.6)$ & $117(8.9)$ & $102(7.7)$ \\
\hline$P$ value & 0.0010 & 0.3505 & 0.0021 & $<0.0001$ & $<0.0001$ \\
\hline
\end{tabular}

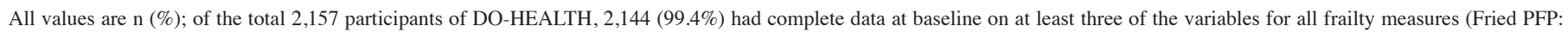

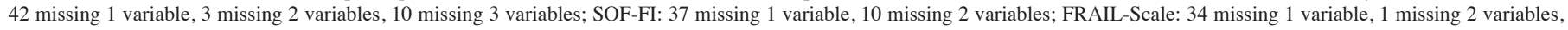

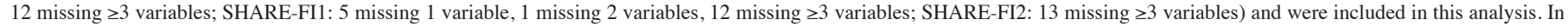

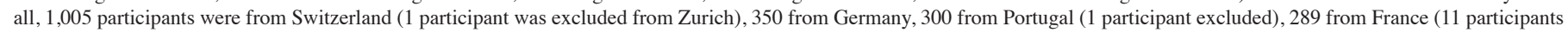
excluded), and 200 from Austria). Fisher's exact test was used to compare subgroups by country. Chi-square tests were used to compare frailty between subgroups by age and gender.

32) (54). Moreover, the observed pattern of a higher frailty prevalence in Portugal is supported by the 2015 OECD data where older adults from southern European countries had worse health states than central European older adults (54).

To the best of our knowledge, we lack population-based data on the prevalence of frailty and pre-frailty for the DO-HEALTH countries comparing different PFP operationalizations with test-based measurements of grip-strength and gait speed (i.e. PFP, SOF-FI) to mainly self-report instruments (i.e. FRAILScale, SHARE-FI). In our analysis, the mainly self-report based instruments indicated a higher frailty prevalence compared with our primary measure PFP that included the test-based measurements of grip-strength and gait speed. This is in contrast to prior research indicating lower levels of frailty by self-report, e.g. in a longitudinal study from Ireland, and thus warrants further investigation (55).

With regard to instruments, the SOF-FI differed most from the PFP in terms of prevalence overall, but also in terms of differences by country, gender, and age. A possible explanation may be that SOF-FI only covers three of the original five PFP components $(56,57)$. A higher prevalence of frailty and prefrailty among Portuguese, female, and participants age 75+ was observed, regardless of the frailty instrument used. Therefore, our findings caution its use as a frailty prevalence tool among relatively healthy older adults.
Our study has several strengths. First, we were able to utilize a large RCT baseline data set of 2,144 meticulously phenotyped older adults with standardized assessments of all participants from five European countries. Second, given the rich DO-HEALTH data set, we were able to match five frailty operationalizations with self-report and test-based assessments derived from Fried's original physical frailty phenotype. Third, despite that fact that DO-HEALTH does not reflect populationbased data and selected relatively healthy older adults, our data provides a conservative comparison of frailty and pre-frailty prevalence by five different frailty operationalizations and between five European countries.

Our study also has its limitations. First, we have to acknowledge that this is not a probabilistic sample and participants were recruited as relatively-healthy volunteers from the regions where the study was performed. Thus, suggesting that the actual prevalence of frailty may be subject to self selection and healthy participant bias (e.g. more frail participants may refrain from participating). Further, adaptions were necessary to apply the original Fried PFP to DO-HEALTH, due to the availability of variables from our data set. However, this has been necessary in most other studies following the initial publication by Fried et al (25, 58). A conceptual limitation of our study is the expected low overall prevalence of frailty in the relatively healthy community 
dwelling older adults enrolled in DO-HEALTH. In addition, DO-HEALTH data does not include information on differences in health care systems or access to primary care between participating centers. However, we still document significant variability between countries, and subgroups.

In summary, our study contributes unique data on the prevalence of pre-frailty and frailty in adults age 70 and older from five European countries enrolled in a large-scale RCT. With regard to the high prevalence of pre-frailty, we add important data indicating that interventions at this early stage possibly modulate the progression to frailty in a substantial share of the older adult population. Thus, our findings may help trigger efforts in the prevention of frailty, not only in Portugal, but also in the central European countries involved in DO-HEALTH. Our results support the fact that fraily prevalence is significantly influenced by the choice of instrument among relatively healthy community dwelling older adults. Further, all instruments but SOF-FI applied in the DO-HEALTH data set, appear to have construct validity with regard to country, gender, and age differences in prevalence of pre-frailty and frailty.

In conclusion, our findings suggest that the choice of frailty instrument among relatively healthy older adults is relevant with regard to frailty prevention and care planning efforts. Given the suggested large health care disparities with regard to frailty among participants from Portugal versus the central European countries in DO-HEALTH, further studies are needed to explore these differences with regard to other aspects of health and at the population-based level.

Funding: This work was supported by the European Commission's 7th framework programme (FP7, HEALTH.2011.2.2.2-1) for investigator-driven clinical trials for therapeutic interventions in elderly populations (Project No: 278588-2). Open access funding provided by University of Zurich.

Acknowledgements: MG drafted the manuscript and interpeted the data. POCB and LAA analysed and interpreted the data and contributed to the drafting of the manuscript. GF and AS provided critical revision of the manuscript. JAK, RWK, BV, JAPD, $\mathrm{DF}, \mathrm{RR}, \mathrm{MB}, \mathrm{EJO}, \mathrm{AE}$ and HABF designed the study concept, aquised the data and critically revised the manuscript. We would like to thank all DO-HEALTH investigators, researchers, and study personnel at the seven partner sites throughout Europe for their contribution.

Conflict of Interest: The authors declare no conflict of interest in regard to this work.

Ethical standards: The DO-HEALTH study was approved by ethics and regulatory agencies of all 5 countries and the study protocol has been previously published (30). A data and safety monitoring board oversaw the study.

Open Access: This article is distributed under the terms of the Creative Commons Attribution 4.0 International License (http://creativecommons.org/licenses/by/4.0/), which permits use, duplication, adaptation, distribution and reproduction in any medium or format, as long as you give appropriate credit to the original author(s) and the source, provide a link to the Creative Commons license and indicate if changes were made.

\section{References}

1. Hoogendijk, E.O., et al., Frailty: implications for clinical practice and public health The Lancet, 2019. 394(10206): p. 1365-1375.

2. Landi, F., et al., Sarcopenia as the Biological Substrate of Physical Frailty. Clin Geriatr Med, 2015. 31(3): p. 367-74.

3. Cruz-Jentoft, A.J., et al., Sarcopenia: revised European consensus on definition and diagnosis. Age and Ageing, 2018: p. afy169-afy169.

4. Verlaan, S., et al., High Prevalence of Physical Frailty Among Community-Dwelling Malnourished Older Adults-A Systematic Review and Meta-Analysis. J Am Med Dir Assoc, 2017.

5. Vanpottelbergh G, Vaes B, Adriaensen W, Mathei C, Legrand D, Wallemacq P, Degryse JM: The glomerular filtration rate estimated by new and old equations as a predictor of important oucomes in elderly patients. BMC Medicine 2014, 12(27):doi:10.11186/11741

6. Rockwood, K. and A. Mitnitski, Frailty Defined by Deficit Accumulation and Geriatric Medicine Defined by Frailty. Clinics in Geriatric Medicine, 2011. 27(1): p. 17-26.

7. Pacifico, J., et al., Prevalence of sarcopenia as a comorbid disease: A systematic review and meta-analysis. Experimental Gerontology, 2020. 131: p. 110801.

8. Fimognari, F.L., et al., The Severity of Acute Illness and Functional Trajectories in Hospitalized Older Medical Patients. The Journals of Gerontology Series A Biological Sciences and Medical Sciences, 2017. 72(1): p. 102-108.

9. Lin, H.S., et al., Frailty and post-operative outcomes in older surgical patients: a systematic review. BMC Geriatr, 2016. 16(1): p. 157.

10. Wou, F., et al., The predictive properties of frailty-rating scales in the acute medical unit. Age Ageing, 2013. 42(6): p. 776-81.

11. Apóstolo, J., et al., Predicting risk and outcomes for frail older adults: an umbrella review of frailty screening tools. JBI Database System Rev Implement Rep, 2017. 15(4): p. 1154-1208

12. Vermeiren, S., et al., Frailty and the Prediction of Negative Health Outcomes: A Meta-Analysis. J Am Med Dir Assoc, 2016. 17(12): p. 1163.e1-1163.e17.

13. Gill, T.M., et al., Transitions between frailty states among community-living older persons. Arch Intern Med, 2006. 166(4): p. 418-23.

14. Subra, J., et al., The integration of frailty into clinical practice: preliminary results from the Gerontopole. J Nutr Health Aging, 2012. 16(8): p. 714-20.

15. Oliveira, J.S., et al., Evidence on Physical Activity and the Prevention of Frailty and Sarcopenia Among Older People: A Systematic Review to Inform the World Health Organization Physical Activity Guidelines. J Phys Act Health, 2020: p. 1-12.

16. Faller, J.W., et al., Instruments for the detection of frailty syndrome in older adults: A systematic review. PLoS One, 2019. 14(4): p. e0216166.

17. Theou, O., et al., What do we know about frailty in the acute care setting? A scoping review. BMC Geriatr, 2018. 18(1): p. 139.

18. Morley, J.E., et al., Frailty consensus: a call to action. J Am Med Dir Assoc, 2013. 14(6): p. 392-7.

19. Rodriguez-Manas, L., et al., Searching for an operational definition of frailty: a Delphi method based consensus statement: the frailty operative definition-consensus conference project. J Gerontol A Biol Sci Med Sci, 2013. 68(1): p. 62-7.

20. Fried, L.P., et al., Frailty in older adults: evidence for a phenotype. J Gerontol A Biol Sci Med Sci, 2001. 56(3): p. M146-56.

21. Mitnitski, A.B., A.J. Mogilner, and K. Rockwood, Accumulation of deficits as a proxy measure of aging. ScientificWorldJournal, 2001. 1: p. 323-36.

22. Rockwood, K., A. Mogilner, and A. Mitnitski, Changes with age in the distribution of a frailty index. Mech Ageing Dev, 2004. 125(7): p. 517-9.

23. Buta, B.J., et al., Frailty assessment instruments: Systematic characterization of the uses and contexts of highly-cited instruments. Ageing Res Rev, 2016. 26: p. 53-61.

24. Searle, S.D., et al., A standard procedure for creating a frailty index. BMC Geriatr, 2008. 8: p. 24

25. Theou, O., et al., Modifications to the frailty phenotype criteria: Systematic review of the current literature and investigation of 262 frailty phenotypes in the Survey of Health, Ageing, and Retirement in Europe. Ageing Res Rev, 2015. 21: p. 78-94.

26. Ensrud, K.E., et al., Comparison of 2 frailty indexes for prediction of falls, disability, fractures, and death in older women. Arch Intern Med, 2008. 168(4): p. 382-9.

27. Abellan van Kan, G., et al., The I.A.N.A Task Force on frailty assessment of older people in clinical practice. J Nutr Health Aging, 2008. 12(1): p. 29-37.

28. Santos-Eggimann, B., et al., Prevalence of frailty in middle-aged and older community-dwelling Europeans living in 10 countries. J Gerontol A Biol Sci Med Sci, 2009. 64(6): p. 675-81

29. Manfredi, G., et al., Prevalence of frailty status among the European elderly population: Findings from the Survey of Health, Aging and Retirement in Europe Geriatr Gerontol Int, 2019. 19(8): p. 723-729.

30. Bischoff-Ferrari, H.A., et al., DO-HEALTH: Vitamin D3 - Omega3 - Home exercise - Healthy aging and longevity trial - Design of a multinational clinical trial on healthy aging among European seniors. Contemporary Clinical Trials, 2020: p. 106124

31. Folstein, M.F., S.E. Folstein, and P.R. McHugh, "Mini-mental state". A practical method for grading the cognitive state of patients for the clinician. J Psych Res, 1975 12 .

32. Guralnik, J.M., et al., Lower extremity function and subsequent disability: consistency across studies, predictive models, and value of gait speed alone compared with the short physical performance battery. J Gerontol A Biol Sci Med Sci, 2000. 55(4): p. M221-31.

33. Sangha, O., et al., The Self-Administered Comorbidity Questionnaire: a new method to assess comorbidity for clinical and health services research. Arthritis Rheum, 2003. 49(2): p. 156-63

34. Bouillon, K., et al., Measures of frailty in population-based studies: an overview. BMC Geriatr, 2013. 13: p. 64

35. Sipers, W.M., et al., The Martin Vigorimeter Represents a Reliable and More Practical Tool Than the Jamar Dynamometer to Assess Handgrip Strength in the Geriatric Patient. J Am Med Dir Assoc, 2016. 17(5): p. 466.e1-7.

36. Ensrud, K.E., et al., A comparison of frailty indexes for the prediction of falls, disability, fractures, and mortality in older men. J Am Geriatr Soc, 2009. 57(3): p. 492-8.

37. Kiely, D.K., L.A. Cupples, and L.A. Lipsitz, Validation and comparison of two frailty indexes: The MOBILIZE Boston Study. J Am Geriatr Soc, 2009. 57(9): p. 1532-9.

38. Guralnik, J.M., et al., A short physical performance battery assessing lower extremity 
function: association with self-reported disability and prediction of mortality and nursing home admission. J Gerontol, 1994. 49(2): p. M85-94.

39. Abellan van Kan, G., et al., Frailty: toward a clinical definition. J Am Med Dir Assoc, 2008. 9(2): p. 71-2.

40. Morley, J.E., T.K. Malmstrom, and D.K. Miller, A simple frailty questionnaire (FRAIL) predicts outcomes in middle aged African Americans. J Nutr Health Aging, 2012. 16(7): p. 601-8

41. Vellas, B., et al., Designing pharmaceutical trials for sarcopenia in frail older adults: EU/US Task Force recommendations. J Nutr Health Aging, 2013. 17(7): p. 612-8.

42. Woo, J., et al., Frailty Screening in the Community Using the FRAIL Scale. Journal of the American Medical Directors Association, 2015. 16(5): p. 412-419.

43. Cuenoud, P., Prevalence of the Frailty Phenotype in community-dwelling population aged 50 and over in the Survey of Health, Ageing and Retirement in Europe, in IEMS Lausanne - Faculté des Hautes Etudes Commerciales et Faculté de Biologie et de Médecine. 2007, Université de Lausanne.

44. Romero-Ortuno, R., The SHARE operationalized frailty phenotype: a comparison of two approaches. Eur Geriatr Med, 2013. 4(4).

45. Pialoux, T., J. Goyard, and B. Lesourd, Screening tools for frailty in primary health care: a systematic review. Geriatr Gerontol Int, 2012. 12(2): p. 189-97.

46. Verghese, J., et al., Self-reported difficulty in climbing up or down stairs in nondisabled elderly. Arch Phys Med Rehabil, 2008. 89(1): p. 100-4.

47. Woods, N.F., et al., Frailty: emergence and consequences in women aged 65 and older in the Women's Health Initiative Observational Study. J Am Geriatr Soc, 2005. 53(8): p. 1321-30.

48. Cawthon, P.M., et al., Frailty in older men: prevalence, progression, and relationship with mortality. J Am Geriatr Soc, 2007. 55(8): p. 1216-23.
49. Fuchs, J., M. Busch, and C. Scheidt-Nave, [Frailty: Prevalence in the Resident Population of Germany 70 - 79 Years of Age - a Population-Based Approach]. Anasthesiol Intensivmed Notfallmed Schmerzther, 2017. 52(11-12): p. 758-763.

50. Gomes, M., et al., Physical inactivity among older adults across Europe based on the SHARE database. Age Ageing, 2017. 46(1): p. 71-77.

51. Portela, D., et al., Instrumental Activities of Daily Living (iADL) Limitations in Europe: An Assessment of SHARE Data. Int J Environ Res Public Health, 2020. 17(20).

52. Theou, O., et al., Exploring the relationship between national economic indicators and relative fitness and frailty in middle-aged and older Europeans. Age Ageing, 2013. 42(5): p. 614-9.

53. World Health Organization. World report on ageing and health. 2015 30.04.2017]; Available from: http://apps.who.int/iris/bitstream/10665/186463/1/9789240694811_ eng.pdf?ua $=1$.

54. OECD, Health at a Glance 2015. OECD Publishing.

55. Theou, O., et al., Measuring frailty using self-report and test-based health measures. Age Ageing, 2015. 44(3): p. 471-7.

56. Fried, L.P., et al., Nonlinear multisystem physiological dysregulation associated with frailty in older women: implications for etiology and treatment. J Gerontol A Biol Sci Med Sci, 2009. 64(10): p. 1049-57.

57. Bandeen-Roche, K., et al., Phenotype of frailty: characterization in the women's health and aging studies. J Gerontol A Biol Sci Med Sci, 2006. 61(3): p. 262-6.

58. Saum, K.U., et al., Development and evaluation of a modification of the Fried frailty criteria using population-independent cutpoints. J Am Geriatr Soc, 2012. 60(11): p 2110-5.

How to cite this article: M. Gagesch, P.O. Chocano-Bedoya, L.A. Abderhalden, et al. Prevalence of Physical Frailty: Results from the DO-HEALTH Study. J Frailty Aging 2022;11(1)18-25; http://dx.doi.org/10.14283/jfa.2021.18 